\title{
Efecto del control de malezas sobre el prendimiento y crecimiento inicial de plantaciones de Pinus ponderosa en la Patagonia Argentina
}

\author{
Effects of weed control on survival and early growth of Pinus ponderosa plantations \\ in Patagonia, Argentina
}

\author{
${\text { Miguel Davel }{ }^{*} \text {, Luis Tejera }}^{2}$, Martín Honorato² ${ }^{2}$ Eduardo Sepúlveda $^{1-3}$ \\ *Autor de correspondencia: ${ }^{1}$ Centro de Investigación y Extensión Forestal Andino Patagónico (CIEFAP). \\ CC14, CP9200, Esquel, Chubut, Argentina, Tel./Fax: +54 2945 453948, mdavel@ ciefap.org.ar \\ ${ }^{2}$ Instituto Nacional de Tecnología Agropecuaria (INTA), Esquel, Argentina. \\ ${ }^{3}$ Consejo Agrario Provincial de la Provincia de Santa Cruz, Los Antiguos, Santa Cruz, Argentina.
}

\begin{abstract}
SUMMARY
Pinus ponderosa is the tree species with the highest potential for plantation in the Andean Patagonian Region of Argentina. Today, most of these plantations are carried out without any site preparation nor taking into account the relationship site-type of seedling. In this study we present the results of three plantation trials carried out in three different sites (regarding precipitation regime and vegetational characteristics), by using two types of seedlings, and with and without herbicide weed control. The period analyzed corresponds to 6 years after trial installation. A differential response was found among types of plant growing in the different sites, and an increase in establishment and growth was registered for those plants grown in weed-free areas when compared to the untreated controls. It was also shown that the herbicide effect upon plant growth starts appearing the second year after treatment.
\end{abstract}

Key words: Patagonia, ponderosa pine, plantation, types of seedlings, herbicide.

RESUMEN

Pinus ponderosa es la especie implantada con mayor potencial en la región andino patagónica en Argentina. Actualmente las plantaciones se realizan sin ninguna preparación del sitio y sin tener en cuenta la relación sitio-tipo de planta. En este trabajo se presentan los resultados de tres ensayos de plantación, instalados en tres sitios distintos en cuanto a precipitación y vegetación, utilizando dos tipos de planta y con y sin control de malezas con herbicidas. El período analizado corresponde a los seis años posteriores a la instalación de los ensayos. Se encontró un comportamiento diferencial de los tipos de planta en los diferentes sitios y un aumento importante en prendimiento y crecimiento inicial en altura, eliminando las malezas mediante el uso de herbicidas. Además se observó que el efecto de la eliminación de malezas sobre el crecimiento comienza a manifestarse recién al segundo año.

Palabras clave: Patagonia, pino ponderosa, plantación, tipos de plantines, herbicida.

\section{INTRODUCCIÓN}

La plantación es una de las tareas más importantes y que implica mayores inversiones para el establecimiento de un bosque. El éxito de un turno completo a menudo está determinado por la adecuación de las decisiones tomadas en el momento de la plantación. Los errores cometidos aumentan generalmente la cantidad de tratamientos posteriores y puede ocurrir que éstos no sean corregidos completamente durante el proceso (Hawley y Smith 1982).

Daniel et al. (1982) indican la importancia del tamaño de las plantas y su relación con las condiciones del sitio. En sitios donde la humedad no es una limitante severa, las plantas más grandes tendrán mayor supervivencia y más rápido crecimiento que las plantas pequeñas. En cambio en sitios secos y ventosos, donde las plantas pueden sufrir un alto estrés hídrico y la inhabilidad para retener agua es la causa más común de mortalidad, las plantas chicas son más adecuadas por su menor relación parte aérea/sistema radicular y su mayor crecimiento inicial de raíz. Además las plantas altas son más adecuadas para lugares en los cuales existe competencia de malezas o el daño de animales puede ser severo (Mexal y Landis 1990).

Algunos autores indican que el efecto del tamaño inicial de las plantas en el crecimiento posterior a la plantación parece ser independiente del modo en que 
esas plantas fueron cultivadas con el fin de alcanzar un tamaño específico. En este sentido Mellberg y Naslund (citados por Mexal y Landis 1990) encontraron que el crecimiento en altura, evaluados 15 años de crecimiento en plantas de scots pine (Pinus sylvestris L.) y norway spruce (Picea abies (L.) Karst.) de diferentes tipos, estuvo estrechamente relacionado a la altura inicial de las plantas, plantines $1+0$ tenían igual potencial de crecimiento que plantines $2+0$ de la misma altura inicial.

La competencia de las malezas muchas veces afecta negativamente la sobrevivencia y el crecimiento, en especial en el primer año de vida de las plantaciones. Deyoe (1986) indica que varios estudios han demostrado que el control de la vegetación competidora durante los 2 a 4 primeros años de la plantación puede duplicar la producción en volumen de los plantines en buenos sitios y al menos triplicarla en los sitios medios a pobres. Powers et al. (1992) reportan que la aplicación de herbicidas y fertilizantes incrementó hasta siete veces la velocidad de crecimiento en plantaciones de pino ponderosa (Pinus ponderosa Dougl. ex Laws) para ocho sitios de USA. Estos efectos también fueron encontrados para pino radiata (Pinus radiata D. Don) en Chile por Hermosilla y Martino (1982), quienes mencionan crecimientos en volumen de cuatro veces superiores en las parcelas donde se eliminaron las malezas con herbicidas. En plantaciones de esta misma especie, Gerding et al. (1986), en Chile, observaron mayor vigorosidad, crecimiento en altura y diámetro de copa, con la aplicación combinada de herbicidas y fertilizantes. En cuanto al prendimiento, Heidmann (1987) encontró que la supervivencia en dos plantaciones de pino ponderosa, en sitios cubiertos por vegetación herbácea, fue de 94 y 99\% cuando la pastura fue muerta con herbicida y menciona que el alto prendimiento se debería no sólo a la eliminación del pasto sino también a la mayor retención de humedad debida a la cubierta formada por éstos. En un estudio anterior, el mismo autor determinó que la humedad del suelo en los primeros $20 \mathrm{~cm}$ fue dos veces más alta bajo los pastos muertos luego de la aplicación de herbicida que bajo los mismos pastos vivos. Además la humedad del suelo bajo la vegetación muerta fue un tercio más alta que en parcelas donde la misma fue extraída en forma manual.

El pino ponderosa es la especie introducida con mayor potencial en la región patagónica. En la actualidad las forestaciones se realizan sin ninguna preparación del sitio y sin tener en cuenta el tamaño de las plantas y su relación con las características del sitio a forestar. Con el presente trabajo se pretende realizar una evaluación de estos dos importantes factores: tipo de planta a utilizar y eliminación de malezas mediante el uso de herbicidas, con el objetivo de lograr un aumento en el prendimiento y en el crecimiento inicial de las plantaciones de pino ponderosa en la región patagónica.

\section{MÉTODOS}

Área de estudio. Los ensayos se ubicaron en el área de potencial forestal para la especie en la provincia del Chubut. La misma se extiende entre $\operatorname{los} 42^{\circ}$ y $44^{\circ}$ de latitud sur y entre los 400 y $1.000 \mathrm{~mm}$ de precipitación media anual. Para la selección de estos sitios se tuvo en cuenta este último factor por ser el de mayor variación en la región y el que origina un fuerte gradiente edáfico y vegetacional en sentido longitudinal. Los tres sitios seleccionados presentan precipitaciones medias anuales de aproximadamente 400, 600 y $1.000 \mathrm{~mm}$.

Descripción de los sitios de ensayo. En cada lugar donde se instalaron los ensayos se recopiló información climática (precipitación media anual), topográfica (altura sobre el nivel del mar, exposición del terreno, pendiente, latitud y longitud) y edáfica (profundidad total y textura) (cuadro 1). Como se puede observar, por el valor de precipitación y la exposición, el sitio 1 es el más extremo para la especie. Los otros dos ensayos se encuentran en sitios de productividad media a alta. Los tres sitios presentaron una pendiente suave de 2 a $4 \%$.

Cuadro 1. Características climáticas, topográficas y edáficas de los tres sitios de ensayo.

Climatic, topographic and edaphic characteristics of the three trial sites.

\begin{tabular}{|c|c|c|c|c|c|c|c|c|}
\hline Ensayo & $\begin{array}{l}\text { Precipitación } \\
\text { media anual } \\
(\mathrm{mm})\end{array}$ & $\begin{array}{l}\text { Altitud } \\
\text { (m snm) }\end{array}$ & Longitud & Latitud & Exposición & $\begin{array}{l}\text { Profundidad } \\
\text { efectiva del } \\
\text { suelo }(\mathrm{m})\end{array}$ & Textura & $\begin{array}{c}\text { Cobertura } \\
\text { herbácea } \\
(\%)\end{array}$ \\
\hline 1 & 395 & 750 & $71^{\circ} 15^{\prime} 06^{\prime \prime}$ & $42^{\circ} 54^{\prime} 37^{\prime \prime}$ & $\mathrm{O}-\mathrm{SO}$ & 0,9 & franco & 60 \\
\hline 2 & 575 & 760 & $71^{\circ} 18^{\prime} 47^{\prime \prime}$ & $42^{\circ} 55^{\prime} 56^{\prime \prime}$ & $S-S E$ & 0,9 & franco & 40 \\
\hline 3 & 990 & 476 & $71^{\circ} 32^{\prime} 40^{\prime \prime}$ & $43^{\circ} 06^{\prime} 32^{\prime \prime}$ & $\mathrm{E}$ & $>1$ & $\begin{array}{c}\text { franco } \\
\text { arenoso }\end{array}$ & 70 \\
\hline
\end{tabular}


También se identificó a la vegetación en tres estratos: herbácea, arbustiva y arbórea. De cada uno de estos estratos se tomó la siguiente información: especies, altura promedio y $\%$ de cobertura. En los ensayos 1 y 2 la vegetación competidora fue herbácea y con una altura de entre 0,5 a $1 \mathrm{~m}$ en el ensayo 1 y de $0,5 \mathrm{~m}$ en el ensayo 2 . En ambos sitios estaba representada por las siguientes especies: neneo (Mulinum spinosum Pers.), coirón (Stipa spp.), senecio (Senecio spp.), pimpinela (Acaena pinnatifida Ruiz et Pavón), Agropyron spp. Mientras que el ensayo 3 presentó vegetación herbácea (70\%) y arbustiva (10\%), predominando cardo (Carduus spp.), Geranium sessiliflorum Cav., Poa spp., diente de león (Taraxacum officinale Weber), ajenjo (Artemisia absinthium L.), retamo (Diostea juncea (Gillies ex Hook.) Miers), radal (Lomatia hirsuta (Lam.) Diles ex J. F. Macbr.), laura (Schinus patagonicus (Phil.) I. M. Johnst.) y calafate (Berberis buxifolia Lam.). En este caso la vegetación herbácea tenía una altura que no sobrepasaba los $0,60 \mathrm{~m}$ y la vegetación arbustiva presentaba entre 1 y $4 \mathrm{~m}$ de altura. Los sitios 1 y 3 fueron los que presentaron mayor cobertura de vegetación (cuadro 1).

Diseño de los ensayos. Se utilizó un diseño factorial completamente al azar, siendo los factores el tipo de planta y la eliminación de malezas mediante la aplicación de herbicida. Los tratamientos aplicados fueron los siguientes:

1+1T : plantas con un año de almácigo y uno de repique $(1+1)$, testigo (sin eliminación de malezas).

$1+2 \mathrm{~T}$ : plantas con un año de almácigo y dos de repique $(1+2)$, testigo.

$1+1 \mathrm{H}$ : plantas $1+1$ con aplicación de herbicida.

$1+2 \mathrm{H}$ : plantas $1+2$ con aplicación de herbicida.
Se realizaron tres repeticiones por tratamiento. Cada parcela incluyó 36 plantas que se plantaron a un distanciamiento de $1 \mathrm{~m}$ x 1m. Se utilizaron plantas 1+1 (un año de almácigo y uno de repique) y 1+2 (un año de almácigo y dos de repique) producidas a raíz desnuda en viveros de la zona y con semilla local. Las plantas fueron seleccionadas en vivero teniendo en cuenta tamaño, vigor, estado sanitario, desarrollo radical y diámetro de cuello, descartándose las plantas muy pequeñas, con tallo débil no lignificado, bifurcadas, torcidas, con raíces enruladas o curvadas y las que presentaban daños, clorosis o síntomas de ataque o enfermedad. A las plantas seleccionadas se les efectuó una poda de raíz, llevándose al campo con las raíces embarradas y protegidas de la desecación.

La plantación fue realizada por dos operarios, utilizando pala de punta. Esta actividad se realizó a más tardar al día siguiente de extraídas las plantas del vivero. De cada uno de los dos tipos de plantas llevados a plantación, se extrajo una muestra al azar de 40 plantas para describir la calidad del material utilizado (largo total, longitud y peso seco de la parte aérea y del sistema radical y diámetro a la altura del cuello) (Davel et al. 1995) (cuadro 2).

Las plantas $1+2$ eran de mayor tamaño que las $1+1$, principalmente en la parte aérea y en el diámetro de cuello, pero eran más desbalanceadas en su relación parte aérea/sistema radicular (cuadro 2).

Para evitar la pérdida de plantas por ataque de liebres, se instaló un alambre tejido perimetral en cada uno de los ensayos, esto se hizo antes de realizar la plantación y una vez marcadas las parcelas en el terreno con estacas.

Cuadro 2. Mediciones realizadas en la muestra de plantas $(1+1$ y $1+2)$ utilizadas para describir la calidad del material utilizado en cada ensayo.

Measurements made in the sampling of seedlings $(1+1$ y $1+2)$ used to describe the quality of the material used in each trial.

\begin{tabular}{|c|c|c|c|c|c|c|c|c|c|c|c|c|c|}
\hline \multirow{2}{*}{$\begin{array}{l}\text { Ensayo/ } \\
\text { Tipo de } \\
\text { planta }\end{array}$} & \multirow[t]{2}{*}{$\mathrm{n}$} & \multicolumn{2}{|c|}{$\begin{array}{l}\text { Largo parte } \\
\text { aérea }(\mathrm{cm})\end{array}$} & \multicolumn{2}{|c|}{$\begin{array}{l}\text { Largo raíz } \\
\qquad(\mathrm{cm})\end{array}$} & \multicolumn{2}{|c|}{$\begin{array}{l}\text { Diámetro de } \\
\text { cuello }(\mathrm{mm})\end{array}$} & \multicolumn{2}{|c|}{$\begin{array}{l}\text { Peso seco } \\
\text { parte aérea } \\
(\mathrm{PSA})(\mathrm{g})\end{array}$} & \multicolumn{2}{|c|}{$\begin{array}{l}\text { Peso seco raíz } \\
\quad(\text { PSR) (g) }\end{array}$} & \multicolumn{2}{|c|}{ PSA/PSR } \\
\hline & & Media & $\begin{array}{l}\text { Desv. } \\
\text { Est. }^{(1)}\end{array}$ & Media & $\begin{array}{l}\text { Desv. } \\
\text { Est. }\end{array}$ & Media & $\begin{array}{c}\text { Desv. } \\
\text { Est. }\end{array}$ & Media & $\begin{array}{l}\text { Desv. } \\
\text { Est. }\end{array}$ & Media & $\begin{array}{l}\text { Desv. } \\
\text { Est. }\end{array}$ & Media & $\begin{array}{c}\text { Desv. } \\
\text { Est. }\end{array}$ \\
\hline $1 / 1+1$ & 40 & 13,0 & 2,2 & 22,1 & 2,6 & 5,0 & 0,7 & 3,8 & 1,4 & 1,7 & 0,6 & 2,2 & 0,4 \\
\hline $1 / 1+2$ & 40 & 21,7 & 3,4 & 23,6 & 2,7 & 7,2 & 1,1 & 11,8 & 4,0 & 3,3 & 1,1 & 3,6 & 0,7 \\
\hline $2 / 1+1$ & 35 & 10,2 & 1,2 & 22,9 & 1,6 & 5,1 & 0,6 & 3,9 & 1,1 & 2,0 & 0,6 & 1,9 & 0,3 \\
\hline $2 / 1+2$ & 35 & 21,7 & 2,9 & 22,2 & 1,9 & 6,8 & 0,9 & 13,4 & 3,1 & 3,3 & 0,8 & 4,1 & 0,7 \\
\hline $3 / 1+1$ & 35 & 9,6 & 1,4 & 20,9 & 2,0 & 3,4 & 0,5 & 2,0 & 0,9 & 1,0 & 0,4 & 1,9 & 0,4 \\
\hline $3 / 1+2$ & 35 & 19,1 & 2,9 & 24,5 & 2,2 & 6,6 & 0,9 & 10,7 & 3,7 & 3,2 & 1,0 & 3,4 & 0,5 \\
\hline
\end{tabular}

(1) Desv. Est: desviación estándar. 
El herbicida utilizado fue de acción total (glifosato). El mismo se aplicó en un círculo de $1 \mathrm{~m}$ de diámetro alrededor de cada planta. La aplicación del herbicida se realizó en la primavera siguiente a la plantación, entre los meses de octubre y noviembre, una vez comenzada la brotación de las malezas y en días calmos, sin viento. Para la aplicación se utilizaron mochilas pulverizadoras de 12 y 22 litros, con punta de diámetro regulable, orificio circular y proyección en abanico. Debido a la no selectividad del herbicida se debió proteger a las plantas cubriéndolas con bolsas de nylon. La dosis utilizada fue de 480 g p.a. por cada 100 litros de agua (1 litro del herbicida por cada 100 litros de agua).

Toma y análisis de los datos. Al momento de la plantación y al finalizar el primero, segundo y sexto año se realizó la toma de datos para la evaluación del prendimiento (porcentaje de plantas vivas) y crecimiento en altura para cada tratamiento. Las mediciones se realizaron en el mes de abril, luego de pasado el periodo de crecimiento correspondiente. Para ello se realizó el conteo de plantas vivas por parcela y la medición de la altura total de cada planta utilizando un metro de madera, obteniéndose los valores promedio por parcela.

Cada sitio fue analizado independientemente. Para determinar la existencia de diferencias significativas entre los distintos tratamientos, se realizó un análisis de varianza y para la comparación de medias de tratamientos se utilizó la prueba de Tukey.

Como información adicional, durante el primer año en el período seco (noviembre-marzo) y una vez al mes, se extrajeron muestras de suelo entre los 20 y $30 \mathrm{~cm}$ de profundidad. Estas muestras se utilizaron para comparar el contenido de humedad de las parcelas donde se aplicó herbicida con las testigos. La determinación del contenido de humedad se realizó mediante la técnica gravimétrica. Primeramente se determinó el peso húmedo $(\mathrm{PH})$ de una muestra, luego se secó a estufa a $105^{\circ} \mathrm{C}$ hasta peso constante (PS) y por último se calculó el contenido de humedad (en porcentaje) con la ecuación [1]:

$$
((\mathrm{PH}-\mathrm{PS}) / \mathrm{PS}) * 100
$$

\section{RESULTADOS}

Ensayo 1 (sitio con menor precipitación). Los resultados obtenidos en este ensayo, en prendimiento y crecimiento en altura, se presentan en la figura 1 , donde se observó un mejor comportamiento de las plantas más chicas $(1+1)$ con respecto a las plantas grandes $(1+2)$, tanto en las parcelas testigo como en las que se eliminó la vegetación competidora. Para ambos tipos de planta se observó una mayor sobrevivencia y un mayor crecimiento en altura al eliminar las malezas mediante el uso de herbicidas.
El análisis estadístico, en el primer año, no mostró diferencias significativas entre tipos de planta $(P=0,606)$ ni entre tratamientos $(P=0,606)$, en cuanto al crecimiento en altura. Sí se observaron diferencias significativas a partir del segundo año y en el sexto año. Estas diferencias, que fueron en aumento del primero al sexto año, fueron debidas a la eliminación de la competencia de malezas mediante el uso de herbicidas $(P=0,0003)$. No se observaron diferencias significativas entre tipos de planta $(P=0,07)$ ni interacción entre tipo de planta y tratamiento $(P=0,73)$.

En cuanto al prendimiento se observaron diferencias significativas desde el primer año, entre tipos de planta a favor de las plantas $1+1(P=0,0004) \mathrm{y}$, entre tratamientos, a favor de la eliminación de malezas con herbicidas $(P=0,0004)$. En ningún caso hubo interacción entre tipo de planta y tratamiento, aunque el valor $P=0,06$ muestra una tendencia que debería seguirse evaluando.

En base a los resultados obtenidos, en el sitio más extremo en lo que respecta a precipitación y exposición al viento para esta especie, los mejores comportamientos luego de seis años de evaluación correspondieron a las plantas $1+1$, tanto en prendimiento como en crecimiento. Para los dos tipos de planta se observó un efecto importante de la eliminación de malezas con el herbicida sobre el prendimiento y sobre el crecimiento, este último efecto se manifestó recién al segundo año.

Ensayo 2 (sitio intermedio). En la figura 1 se puede observar que no hubo diferencias de prendimiento entre los dos tipos de planta. Sí hubo diferencias, para ambos casos, con la aplicación de herbicidas, donde se lograron prendimientos de entre 10 y $12 \%$ superiores al testigo. Las diferencias observadas fueron inferiores a las del ensayo anterior y éstas se pueden deber a la mayor precipitación, la menor cobertura de la vegetación competidora y a la mayor retención de agua del suelo en este ensayo. Al sexto año, si bien en todos los casos hubo un aumento de la mortalidad con respecto al primer año, este fue más notorio en las plantas $1+1$ sin aplicación de herbicida.

En cuanto al crecimiento en altura no se observaron diferencias importantes, en el primer año, entre tipos de planta ni entre tratamientos. A partir del segundo año y hasta el sexto, las diferencias entre tipos de planta se mantuvieron pero se hicieron más notorias las diferencias entre tratamientos de las parcelas con aplicación de herbicidas con respecto a las parcelas testigo. El mejor comportamiento se observó en las plantas $1+2$ y eliminando las malezas mediante el uso de herbicidas.

El análisis de varianza no mostró diferencias significativas en prendimiento, entre tipos de planta $(P=0,72)$ ni entre tratamientos $(P=0,07)$, para los dos primeros períodos de crecimiento considerados, aunque el valor de $\mathrm{p}$ entre tratamientos muestra una tendencia. Sin embargo, al sexto año y debido al aumento de la mortalidad de 

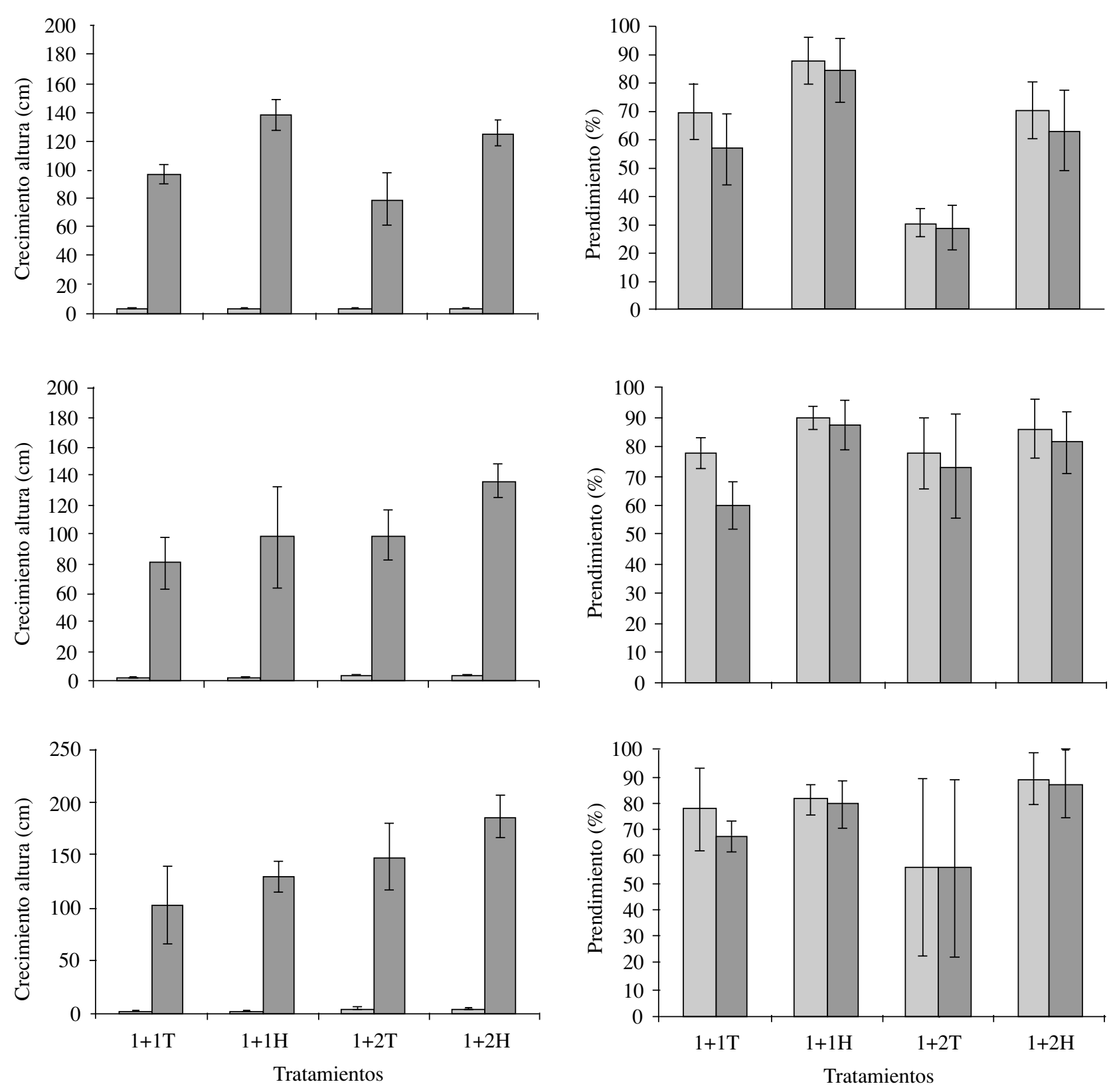

Año 1

Año 6

Figura 1. Crecimiento en altura y prendimiento al año y luego de 6 años de la plantación, para los distintos tratamientos en los tres ensayos.

Height growth and survival at one and six years after plantation for the different treatments in the three trials.

plantas en las parcelas testigo, las diferencias de prendimiento se hicieron significativas a favor de las parcelas tratadas con herbicida $(P=0,03)$.

En cuanto al crecimiento en altura, el análisis estadístico indicó que las diferencias observadas al sexto año fueron significativas entre tipos de planta a favor de las plantas $1+2(P=0,05)$.

La evaluación del porcentaje de humedad durante el período seco mostró que los mayores valores se presen- taron en las parcelas donde se eliminó la vegetación competidora (figura 2).

Ensayo 3 (sitio de mayor precipitación). En el sitio con mayor cobertura de vegetación competidora se pudo ver, en la figura 1, un mayor prendimiento de las plantas $1+2$. Contrariamente a lo esperado, la excepción son las parcelas testigo, donde se observó un mayor prendimiento de las plantas chicas. Para ambos tipos de planta 
el prendimiento aumentó con la eliminación de las malezas con el herbicida, lo que se hace más notorio en las plantas $1+2$.

En cuanto al crecimiento en altura se observó, desde el primer año, un mayor crecimiento de las plantas 1+2, tanto en las parcelas testigo como en donde se aplicó herbicida. El efecto de la eliminación de malezas sobre el crecimiento, como se observó en los demás ensayos, se manifestó en mayor medida a partir del segundo año. En este ensayo esto se hizo bien notorio en las plantas $1+2$.

El análisis estadístico al sexto año mostró diferencias significativas en el crecimiento en altura, entre tipos de planta a favor de las plantas $1+2(P=0,01)$. Estas diferencias se observaron desde el primer periodo de crecimiento luego de la plantación $(P=0,001)$. Las diferencias en crecimiento debidas a los tratamientos resultaron no significativas desde el punto de vista estadístico ni al primero $(P=0,5)$ ni al sexto año $(P=0,07)$, sin embargo en este último el valor observado de $P$ evidenció una tendencia de mayor crecimiento al aplicar herbicidas.

En cuanto al prendimiento no hubo diferencias significativas entre tratamientos $(P=0,07)$ ni entre tipos de planta $(P=0,83)$, durante todo el periodo analizado. No se observó tampoco interacción entre tipo de planta y tratamiento en ningún caso $(P=0,39)$.

En base a lo mencionado para este sitio, los mejores resultados se lograron con las plantas $1+2$ y eliminando la vegetación competidora con herbicida. Las mismas fueron levemente superiores a las $1+1$ en cuanto a los valores de prendimiento y crecimiento en altura. En contra de lo esperado las plantas 1+1 tuvieron mayor prendimiento que las $1+2$ en las parcelas testigo. Si bien las diferencias en prendimiento no fueron significativas según el análisis estadístico, se observó una tendencia que debería analizarse, ya que los prendimientos logrados en las parcelas testigo (50 y $72 \%$ ) no serían aceptados por los planes de promoción a las forestaciones, actualmente existentes en el país. Esto sí se lograría en las parcelas donde se aplicó herbicida (80 y 90\%).

En lo que respecta a la evaluación de los porcentajes de humedad edáfica en parcelas con y sin eliminación de malezas, los resultados se presentan en la figura 2. En la misma se observan los mayores valores de humedad donde se eliminaron las malezas con herbicida.

\section{DISCUSIÓN}

En las tres situaciones evaluadas y para ambos tipos de plantas se observó un aumento importante en prendimiento y crecimiento inicial con la eliminación de la competencia de malezas mediante el uso de herbicidas, coincidiendo con lo encontrado por otros autores en estudios similares (McDonald y Fiddler 1990, Rose y Ket-

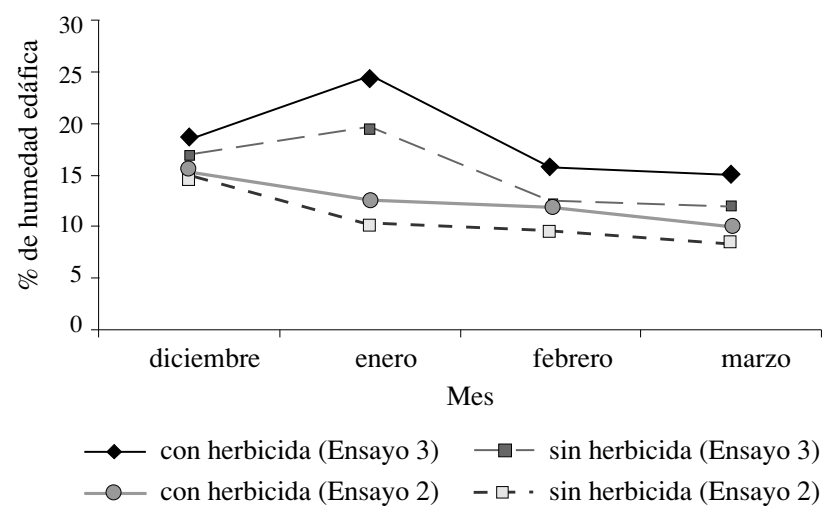

Figura 2. Porcentaje de humedad edáfica entre los 20 y $30 \mathrm{~cm}$ de profundidad, durante el periodo seco del primer año, en las parcelas testigo y donde se eliminó la vegetación competidora, en los ensayos 2 y 3 .

Percentage of edaphic moisture between 20 to $30 \mathrm{~cm}$ of soil depth, during the dry period of the first year, in the control plot and where the competing vegetation was eliminated (trials 2 and 3 ).

chum 2002, Watt et al. 2003, Roberts et al. 2005). Estas diferencias aumentaron en los años siguientes por una mayor mortalidad en las parcelas testigo. Los resultados obtenidos en prendimiento en las parcelas tratadas con herbicida cobran mayor importancia debido al año extraordinariamente seco en que se instalaron los ensayos, donde la precipitación acumulada en el año fue aproximadamente la mitad de la media histórica anual en los tres sitios.

Las diferencias de crecimiento por la eliminación de las malezas se observaron principalmente a partir del segundo año y sobre todo al sexto año. El aumento de las diferencias en el crecimiento con el tiempo coincide con lo observado por Rose y Ketchum (2002) para pino ponderosa y otras coníferas en el noroeste de los Estados Unidos de Norteamérica y por Andenmatten y Letourneau (2002) para pino ponderosa en Patagonia. Estos últimos autores encontraron que la extracción de malezas produjo un aumento en el crecimiento en diámetro y altura de los plantines, pero no observaron diferencias en cuanto a la mortalidad.

Coincidiendo con lo mencionado por Daniel et al. (1982) y Mexal y Landis (1990) hubo un comportamiento diferencial entre los tipos de planta para las distintas condiciones de sitio. En el sitio más seco el mejor comportamiento correspondió a las plantas más chicas $(1+1)$, mientras que en el sitio con mayor precipitación, las plantas más grandes $(1+2)$ fueron las que dieron los mejores resultados. En el sitio intermedio fue donde las diferencias entre tipos de planta fueron menores, aunque con una leve superioridad de las plantas más grandes $(1+2)$. 
La determinación del porcentaje de humedad edáfica durante el periodo seco mostró, en todos los casos, un mayor contenido de humedad en las parcelas tratadas con herbicidas, aunque estas diferencias fueron inferiores a las reportadas por Heidmann (1987) en los Estados Unidos de Norteamérica. Este aspecto es importante para la región debido a que los meses más secos del año coinciden con el periodo de crecimiento de las plantas.

\section{CONCLUSIONES}

- Hubo un comportamiento diferencial del tipo de planta con respecto a las condiciones del sitio. En los sitios más extremos (secos) se comportaron mejor las plantas de menor tamaño $(1+1)$, mientras que en los mejores sitios las plantas más grandes $(1+2)$ tuvieron mayores prendimientos y crecimientos en altura.

- En los tres sitios se obtuvieron importantes ganancias en prendimiento y crecimiento en altura, eliminando la vegetación competidora mediante el uso de herbicidas.

- Las diferencias en el crecimiento en altura entre tipos de planta y por la eliminación de malezas se acentuaron con el paso del tiempo.

- Se observó un mayor contenido de humedad edáfica, durante el periodo seco, donde se eliminaron las malezas con herbicidas. Este es un aspecto importante para la región porque este periodo coincide con la estación de crecimiento de las plantas.

\section{AGRADECIMIENTOS}

A los productores que nos permitieron instalar los ensayos en sus predios. Al CIEFAP, al INTA y a la SAGPyA que financiaron este estudio.

\section{REFERENCIAS}

Andenmatten E, F Letourneau. 2002. Establecimiento y crecimiento inicial de pino ponderosa en la zona subhúmeda a seca de los Andes Patagónicos. Informe Final proyecto
PIA 12/98 del Proyecto Forestal de Desarrollo de la SAGPyA. Argentina. 38 p.

Daniel TW, JA Helms, FS Baker. 1982. Principios de Silvicultura. Ed. McGraw-Hill, México. 492 p.

Davel MM, L Tejera, J Guasp. 1995. Estudio de eficiencia de herramientas de plantación para pino ponderosa en distintos sitios de la provincia del Chubut. In Actas de las IV Jornadas Forestales Patagónicas, S.M. de los Andes. Argentina. Tomo I. p. 253-265.

Deyoe D. 1986. Guidelines for handling seeds and seedlings to ensure vigorous stock. Special Publication 13. College of Forestry, Oregon State University. p. 9-24.

Gerding V, J Schlatter, L Barriga. 1986. Fertilización para el establecimiento de Pinus radiata D. Don en Valdivia. Bosque 7 (2): 121-128.

Hawley RC, DM Smith. 1982. Silvicultura Práctica. Barcelona, España. Omega. p. 242-297.

Heidmann LJ. 1987. Regeneration strategies for ponderosa. In Symposium Proceedings: Ponderosa pine, the species and its management. Spokane, Washington, USA. Editado por Baumgartner DM y JE Lotan. p. 227-233.

Hermosilla M, I Martino. 1982. Influencia del uso de herbicidas, diferentes calidades de plantas y fertilizantes sobre la productividad de un sitio. In Actas Reunión de Trabajo: Evaluación de la productividad de sitios forestales. Universidad Austral de Chile. Valdivia, Chile. p. 244-255.

McDonald P, G Fiddler. 1990. Ponderosa pine seedlings and competing vegetation: ecology, growth and cost. USDA Forest Service Research Paper PSW 199. 10 p.

Mexal JG, TD Landis. 1990. Target Seedling Concepts: height and diameter. Target Seedlings Symposium: Proceedings, combined meeting of the Western Forest Nursery Associations. Oregon, USA. Cap. 3: 17-36.

Powers RF, GT Ferrell, TW Koerber. 1992. The garden of Eden experiment: four year growth of ponderosa pine plantations. Habitat Fiber. USA. 9 p.

Roberts SD, CA Harrington, TA Terry. 2005. Harvest residue and competing vegetation affect soil moisture, soil temperatura, $\mathrm{N}$ availability, and Douglas-fir seedling growth. Forest Ecology and Management 205: 333-350.

Rose R, JS Ketchum. 2002. Interaction of vegetation control and fertilization on conifer species across the Pacific Northwest. Can. J. For. Res. 32: 136-152.

Watt MS, D Whitehead, E Mason, B Richardson, MO Kimberley. 2003. The influence of weed competition for light and water on growth and dry matter partitioning of young Pinus radiata, at a dryland site. Forest Ecology and Management 183: 363-376. 